\title{
Théologiques
}

\section{Les lieux de la théologie aujourd'hui}

\section{Jean Duhaime}

Volume 14, numéro 1-2, automne 2006

Les lieux de la théologie aujourd'hui

URI : https://id.erudit.org/iderudit/014306ar

DOI : https://doi.org/10.7202/014306ar

Aller au sommaire du numéro

Éditeur(s)

Faculté de théologie et de sciences des religions, Université de Montréal

ISSN

1188-7109 (imprimé)

1492-1413 (numérique)

Découvrir la revue

Citer ce document

Duhaime, J. (2006). Les lieux de la théologie aujourd'hui. Théologiques, 14(1-2), 5-8. https://doi.org/10.7202/014306ar

Tous droits réservés $\subset$ Faculté de théologie et de sciences des religions, Université de Montréal, 2006
Ce document est protégé par la loi sur le droit d'auteur. L’utilisation des services d'Érudit (y compris la reproduction) est assujettie à sa politique d'utilisation que vous pouvez consulter en ligne.

https://apropos.erudit.org/fr/usagers/politique-dutilisation/ 


\section{Les lieux de la théologie aujourd'hui}

Jean DuHAIME

Faculté de théologie et de sciences des religions

Université de Montréal

Le colloque dont nous publions ici la plupart des communications, revues par leurs auteurs, est un des événements que nous avons mis sur pied dans le cadre des activités du $125^{\mathrm{e}}$ anniversaire de l'Université de Montréal, dont la Faculté de théologie et de sciences des religions est une des facultés fondatrices. Le $125^{\mathrm{e}}$ anniversaire de la Faculté nous offrait une occasion exceptionnelle d'explorer la situation actuelle de la théologie et sa place dans l'université, l'Église et la société. Tel est le but du colloque que nous avons tenu du 11 au 13 mars 2004.

Dans les sociétés occidentales de tradition chrétienne, la théologie est l'une des disciplines fondatrices de l'université. En interaction constante avec la culture, elle explore les sources et les manifestations historiques du christianisme, sa situation actuelle, ses rapports aux autres traditions religieuses. Elle participe à la vie intellectuelle, aux questionnements éthiques et aux quêtes spirituelles des Églises et des sociétés.

De nos jours, la théologie chrétienne est souvent remise en question. En milieu universitaire, elle apparaît à certains comme un vestige inutile d'une époque révolue où la religion prétendait régir tous les domaines du savoir; considérée comme une activité non-scientifique, ne devrait-elle pas être évacuée de l'université publique?

Les instances ecclésiales se montrent parfois méfiantes à son égard à cause des distances qu'elle semble prendre par rapport à un héritage dogmatique et éthique dont l'affirmation publique se fait plus insistante.

Dans le domaine social et culturel, l'effacement marqué des institutions religieuses et la diversité des formes que prennent les quêtes spirituelles et éthiques de nos contemporains amènent à s'interroger sur la pertinence du patrimoine chrétien.

Comment se positionner face à ces interrogations? Quelles sont la signification et la contribution de la théologie chrétienne dans le monde d'aujourd'hui ? Peut-elle revendiquer encore sa place dans l'université 
moderne et à quelles conditions? Les Églises ont-elles besoin d'une théologie académique? La théologie est-elle concernée par les débats sociaux et politiques actuels? Telles sont les questions que nous souhaitions approfondir. Ce colloque s'inscrit dans une démarche de réflexion que plusieurs facultés et départements de théologie mènent depuis quelques années, principalement dans la francophonie canadienne et européenne. Cet événement réunissait donc des chercheurs du Canada, de Belgique, de France et de Suisse, provenant d'institutions qui se sont penchées sur ces problèmes récemment et dont les idées méritent d'être mises en commun. La démarche proposée s'articule autour de quatre textes principaux.

Jean-Claude Petit, professeur à l'Université de Montréal, amorce la discussion en dressant un état des lieux plutôt sombre. On constate un déclin des inscriptions dans les principales Facultés de théologie et de sciences des religions, au Québec et ailleurs en Occident. Les maisons d'édition publient de moins en moins en théologie, faute d'acheteurs. Le champ théologique s'est fractionné de manière débridée par une multiplication des sujets et des approches. On en vient à se demander «si la prétention chrétienne [...] a perdu [...] pour nos contemporains sa force d'interpellation ». Dans un tel contexte, certains favorisent une action de repli sur l'institution, tandis que d'autres prônent une transformation de la théologie en sciences des religions. Jean-Claude Petit récuse ces deux scénarios. Il rappelle que l'objet essentiel de la théologie est la question de Dieu et que "l'horizon du travail et de la réflexion théologiques [...] est le monde de tous », comme l'écrit Pierre Gisel. S'inspirant de Fernand Dumont, il invite les théologiens à s'ouvrir à la totalité de l'expérience humaine telle qu'elle se présente dans la culture contemporaine.

Faisant part de son expérience comme professeur et ex-doyen de la Faculté de théologie de l'Université catholique de Louvain, Jean-Marie Sevrin soutient que la théologie "peut s'inscrire comme une science humaine dans le champ universitaire avec profit pour elle-même et pour l'université ». En plus de s'intéresser à l'analyse de la "mémoire culturelle » de nos sociétés, la théologie "peut constituer, dans l'université, un pôle de gratuité et de sens » qui met en garde contre l'absolutisation de la rationalité scientifique et technique et se prête particulièrement bien à des démarches interdisciplinaires non seulement avec d'autres sciences humaines (philosophie, histoire, anthropologie, etc.), mais aussi avec les sciences de la nature et de la santé.

Ex-président du Sénat de l'Université de Lausanne, spécialiste de l'histoire du protestantisme et formateur d'adultes dans l'Église réformée de 
Neuchâtel pendant plusieurs années, Denis Müller souligne que le théologien confessionnel œuvrant dans une université publique est dans une position ambivalente: il est à la fois rattaché à son Église et indépendant d'elle. La «loyauté critique» qu'il exerce envers cette institution est souvent incomprise des autorités religieuses, tandis qu'elle paraît suspecte à ses collègues de l'université. Sa condition n'est pourtant pas exceptionnelle, car d'autres champs disciplinaires (droit, génie, médecine, etc.) entretiennent le même genre de rapport avec des instances régulatrices.

En dépit de sa perte d'autorité, la théologie chrétienne est toujours une ressource pour penser les questions de société. Responsable des études de doctorat en théologie à l'Institut catholique de Paris, la moraliste Geneviève Médevielle décrit trois modèles d'engagement social chrétien, en prenant comme exemple l'action de l'Église de France dans l'accueil des étrangers illégaux. Dans la ligne d'une "éthique autonome", les évêques de France sont intervenus dans le débat comme tout autre citoyen en se référant à la valeur commune de la fraternité. L'un d'entre eux, rattaché davantage au modèle de «l'éthique de la foi », a pris position ouvertement au nom de la tradition chrétienne, en rappelant qu'elle « commande de servir Dieu dans les pauvres». Des groupes chrétiens qui privilégient l'approche de "l'éthique communautarienne de la foi » ont développé une position contreculturelle, en tension avec certaines valeurs sociales dominantes; c'est à eux qu'ont fait appel les réfugiés musulmans qui ont cherché asile dans des Églises catholiques.

Les interventions de Jean-Marie Sevrin, Denis Müller et Geneviève Médevielle sont suivies respectivement des réactions des professeurs Robert Mager, Daniel Fraikin et Melchior Mbonimpa, qui permettent de prolonger la réflexion sur chacune de ces questions. Les autres textes de ce recueil, présentés en atelier lors du colloque, approfondissent d'autres facettes de ces problèmes, regroupés en trois sections: la théologie universitaire et les mutations culturelles (Marc Dumas, Louis Perron); la théologie entre l'université et l'Église (Maxime Allard, Marc Pelchat, Normand Provencher); la théologie, l'imaginaire public et les défis éthiques (Michel-M. Campbell, Jean-Guy Nadeau, Hubert Doucet).

Les travaux de ce colloque nous offrent une occasion privilégiée d'élargir la discussion sur « les lieux de la théologie » dans la francophonie canadienne, particulièrement québécoise, tout en profitant des expériences européennes rapportées et interprétées par des représentants de quelquesunes des principales institutions francophones où s'élabore la réflexion 
théologique actuelle. On ne peut que s'en réjouir et souhaiter que des rencontres subséquentes permettent de consolider les liens entre les institutions universitaires de théologie, sur les plans national et international, d'associer des représentants d'autres traditions religieuses à la réflexion des théologiens chrétiens et d'explorer les rapports entre la théologie, les sciences des religions et les autres disciplines académiques.

Au nom des organisateurs (Odette Mainville, Jean-Claude Petit et moimême), des intervenants et des participants au colloque, je remercie le bureau des Fêtes du $125^{\mathrm{e}}$ anniversaire de l'Université de Montréal, le Fonds des donateurs de la Faculté de théologie et de sciences des religions et le Conseil de recherches en sciences humaines du Canada dont les contributions financières ont permis la tenue de ce colloque et la publication du présent numéro. Notre reconnaissance va également aux éditions Médiaspaul, qui ont participé à la révision des textes, à Jenna Smith, pour la traduction de plusieurs résumés d'articles, ainsi qu'à Dany Rodier, pour son précieux travail de mise en forme et de révision des articles, à chaque étape du processus éditorial.

Bonne lecture! 